\title{
A Prognostic Model To Predict Survival In Stage III Colon Cancer Patients Based on Histological Grade, Preoperative Carcinoembryonic Antigen Level and the Neutrophil Lymphocyte Ratio
}

\author{
Zhi-Jun Wuxiao ${ }^{1 \&}$, Hai-Yan Zhou ${ }^{1 \&}$, Ke-Feng Wang ${ }^{2,3}$, Xiao-Qin Chen ${ }^{2,3}$, Xin-Bao \\ $\mathrm{Hao}^{1}$, Yan-Da Lu${ }^{1}$, Zhong-Jun Xia ${ }^{2,3 *}$
}

\begin{abstract}
Background: Stage III colon cancer patients demonstrate diverse clinical outcomes. The aim of this study was to develop a prognostic model in order to better predict their survival. Materials and Methods: From 2004 to 2010,548 patients were retrospectively analyzed, among whom 328 were defined as the study group and the remaining 220 served as a validation group. Clinico-pathologic features, including age, gender, histological grade, T stage, number of positive lymph nodes, number of harvest lymph nodes, pretreatment carcinoembryonic antigen (CEA) levels and pretreatment neutrophil lymphocyte ratio (NLR), were collected. Kaplan-Meier survival curves were used to detect prognostic factors and multivariate analysis was applied to identify independent examples on which to develop a prognostic model. Finally, the model was further validated with the validation group. Results: Histological grade $(p=0.002)$, $T$ stage $(p=0.011)$, number of positive lymph nodes $(p=0.003)$, number of harvested lymph nodes $(p=0.020)$, CEA $(p=0.005)$, and NLR $(p<0.001)$ were found as prognostic factors while histological grade [RR(relative risk):0.632,95\% CI (Confidence interval) 0.405 0.985, $p=0.043]$, CEA (RR:0.644, 95\% CI:0.431 0.964, $p=0.033$ ) and NLR (RR:0.384, 95\% CI:0.255 0.580, $p<0.001)$ levels were independent. The prognostic model based on these three factors was able to classify patients into high risk, intermediate and low risk groups $(p<0.001)$, both in study and validation groups. Conclusions: Histological grade, pretreatment CEA and NLR levels are independent prognostic factors in stage III colon cancer patients. A prognostic model based on these factors merits attention in future clinical practice.
\end{abstract}

Keywords: NLR - CEA - colon cancer - biomarker - prognosis - model

Asian Pac J Cancer Prev, 16 (2), 747-751

\section{Introduction}

With approximate one million new cases every year (Barton, 2012), colon cancer is one of the most severe health burdens all over the world. For stage III colon cancer (T1 4N1 2), standard treatment had been established as radical surgery followed by adjuvant chemotherapy (Moertel et al., 1990; Kuebler et al., 2007; Cassidy et al., 2008) since 1990. However, the treatment outcomes are not as desired- about $60 \%$ stage III patients eventually die from tumor relapse (O'Connell et al., 2004; Giraldez et al., 2013) within five years.

Far from a homogeneous patients group, patients with stage III colon cancer in fact harbor subgroups with notably diverse clinical outcomes- since the five year survival rate ranges from $44 \%$ to $83 \%$ (Wong et al.,
2011). What's more, a considerable amount of patients may remain recurrence free even without adjuvant chemotherapy (Wong et al., 2011), as reported by Adlard et al. (2002) that approximate $60 \%$ stage III colon cancer patients cannot get benefit from adjuvant treatment. Hence, the current staging system still leaves room for improvement to better distinguish patients according to their survival (Qiu et al., 2011). It is advisable to search other criteria to further classify patients and optimize the therapeutic approach- which can both improve survival rates in high risk patients and avoid overtreatment in low risk patients.

Efforts have been done in this field. For example, carcino-embryonic antigen (CEA), which is the most applied tumor marker in colon cancer, has been reported as negatively correlated with survival in operable colon 
cancer patients (Takagawa et al., 2008). In recent years, neutrophil lymphocyte ratio (NLR), which is thought as an indicator of systemic inflammation (Chiang et al., 2012), also has been reported as a prognostic factor for poor overall survival (OS) in colon cancer patients (Chiang et al., 2012). What's more, correlations between survival and several of genes or proteins, including KRAS (Ogino et al., 2009), BRAF (Ogino et al., 2012), microsatellite instability (Bertagnolli et al., 2009), cell cycle proteins (Belt et al., 2012) and MMP (Wong et al., 2011), were also observed. However, the available published papers still leave some to be desired. For example, only about $30 \%$ non-metastatic colon cancer patients have elevated serum CEA levels (Peng et al., 2013), which mitigate its weight in predicting survival. Other gene or protein tests are costly and the available evidences are not yet strong enough to ensure their applications in clinical practice.

Given all this, the aim of this study is to build a prognostic model based on routinely clinic-pathological features to predict survival in patients with stage III colon cancer.

\section{Materials and Methods}

\section{Patient selection}

Patients who met the following criterions were selected: 1) patients underwent radical resection for colon cancer at the The Affiliated Hospital of Hainan Medical College and Sun Yat-sen University Cancer Center between 2004 and 2010 and pathologically confirmed as stage III disease according to American Joint Committee on Cancer (AJCC) Cancer Staging Manual; 2) patients whose Eastern Cooperative Oncology Group (ECOG) status $\leq 2$; 3) with available records of blood routine and CEA before surgery; 4) with available follow-up information. The exclusion criteria were 1) patients who accepted emerging surgery; 2) patients who died within three months after surgery; 3) patients who were with a positive surgical margin; 4) patients who accepted chemotherapy before surgery. 548 patients were involved in this study, among which 328 , which were selected from The Affiliated Hospital of Hainan Medical College, were defined as study group, and 220 which were selected from Sun Yat-sen University Cancer Center were defined as validation group.

\section{Treatment protocol}

Eligible patients all had primary colon adenocarcinoma completely resected. The staging was done based on AJCC Cancer Staging Manual. According to NCCN guild-line, all those stage III patients accepted 5-fluorouracil based adjuvant chemotherapy- including FOLFOX and XELOX. When tumor relapsed, treatments were given according to oncologists' decision.

\section{Laboratory measurement of neutrophil, lymphocyte counts and CEA \\ Neutrophil and lymphocyte were contained in blood routine test. The blood routine tests were conducted by Sysmex XE-5000 ${ }^{\mathrm{TM}}$ Automated Hematology System (Shanghai, China). CEA was evaluated using}

electrochemiluminescence with the Roche Elecsys 2010 Chemistry Analyzer (Basel, Switzerland).

\section{Calculation and grouping of NLR and CEA}

NLR was defined as the neutrophil to lymphocyte ratio, and it was categorized into two groups ( $>3$ and $\leq 3$ ) (Chiang et al., 2012). CEA was divided into two groups as $>5$ and $\leq 5$ according to the normal value range in our hospital.

\section{Patients follow-up}

The follow-up include clinical examination, routine blood test, tumor markers test, CT scan and endoscopy. In the first two years after surgery, follow-up were done every three months. From the third to fifth year follow-up were done every six months and once a year from the sixth year. Overall survival (OS) was defined as the time from diagnosis to the date of death or the last date of follow-up, which was conducted at 31st May 2014.

\section{Statistical analysis}

All statistical analyses were performed using SPSS version 13.0. Frequencies and descriptive statistics were used to represent patient characteristics. The survival curves were calculated using the Kaplan-Meier method. Prognostic factors with a $p$ value less than 0.1 were included in multivariate analysis using a Cox proportional hazards model to test independent significance by backward elimination of insignificant explanatory variables. A P value equals to 0.05 or less was considered to be significant.

\section{Results}

\section{Patient characteristics}

There were 328 consecutive patients enrolled in the study group. Age ranged from 21 to 85, with a median age of 55. The median follow-up time was 46.1 months. 222 were still alive and 106 were dead at the last follow up time. The median OS was 43.2 months (range 4.4 to 86.2). The patients' characteristics were shown in Table 1.

\section{Identification of independent prognostic factors}

The Kaplan-Meier method allowed us to explore all characteristics in Table 1and identified the following parameters impacting survival: histological grade $(p=0.002)$, T stage $(p=0.011)$, number of positive lymph node $(p=0.003)$, number of harvest lymph nodes $(p=0.020), \operatorname{CEA}(p=0.005)$, and NLR $(p<0.001)$, as shown in Table 2. Next, all the factors with a $p$ value less than 0.1 were involve in multivariate analysis. Only histological grade [RR (relative risk):0.632, 95\%CI (Confidence interval) $0.405 \sim 0.985, p=0.043$ ], preoperative CEA levels (RR:0.644,95\%CI:0.431 0.964, $p=0.033$ ) and NLR levels (RR:0.384, 95\%CI:0.255 0.580, $p<0.001$ ) retained their statistical influence on survival.

The prognostic model based on the independent prognostic factors

To obtain a more precise prediction on survival, a prognostic model was developed based on the three 
parameters- by assigning each factor 0 or 1 point as shown in (Table 3). The patient's total score was equal to the sum of his scores in the three parameters. Patients with poor differentiated adenocarcinoma and mucinous adenocarcinoma were classified into the same group since they have similar survival rate, as shown in Figure 1. Since only 11 patients scored 3, they were analyzed together with patients who scored 2. Patients who scored 0 were assigned as low risk group, scored 1 were intermediate group, scored 2 and 3 were high risk

Table 1. The Characteristics of the Patients in Study Group and Validation Group

\begin{tabular}{lcc}
\hline Characteristics & Study group & Validation group \\
\hline $\begin{array}{l}\text { XAge } \\
<60 / \geq 60\end{array}$ & $185 / 143$ & $144 / 76$ \\
$\quad$ Gender & $204 / 124$ & $121 / 99$ \\
Male/female & & \\
$\quad$ Location & $126 / 202$ & $85 / 135$ \\
Right/left colon & & \\
$\quad$ T stage & $44 / 218 / 66$ & $26 / 157 / 37$ \\
T1+2/3/4 & & \\
$\quad$ Lymph nodes removed & $125 / 203$ & $81 / 139$ \\
$<12 / \geq 12$ & & $160 / 60$ \\
$\quad$ Positive lymph nodes & $254 / 74$ & $154 / 46 / 20$ \\
$<4 / \geq 4$ & & $106 / 114$ \\
$\quad$ Histology grade & $231 / 69 / 28$ & \\
High and moderate /poor differentiated/ mucinous \\
CEA & $194 / 134$ & $171 / 49$ \\
$\leq 5 />5$ & & \\
$\quad$ NLR & $249 / 79$ & \\
$\leq 3 />3$ & & \\
\hline
\end{tabular}

Table 2. Characteristics Affecting OS in Univariate Analysis

\begin{tabular}{lc}
\hline Characteristics & P value \\
\hline Age & 0.099 \\
Gender & 0.477 \\
Location & 0.359 \\
Histology grade & 0.002 \\
T stage & 0.011 \\
No. of positive lymph nodes & 0.003 \\
No. of harvest lymph nodes & 0.02 \\
CEA & 0.005 \\
NLR & $<0.001$ \\
\hline
\end{tabular}

Table 3. Prognostic Scoring of the Patients

\begin{tabular}{lcc}
\hline $\begin{array}{l}\text { Characteristics } \\
\text { Histology grade }\end{array}$ & $\begin{array}{c}\text { Scored 0 } \\
\text { High and moderate } \\
\text { differentiated }\end{array}$ & $\begin{array}{c}\text { Scored 1 } \\
\text { poor differentiated/ } \\
\text { mucinous }\end{array}$ \\
\hline CEA & $\leq 5$ & $>5$ \\
NLR & $\leq 3$ & $>3$ \\
\hline
\end{tabular}

Table 4. OS in Patients CLassified by the Prognostic Model

\begin{tabular}{lccc}
\hline Risk group & Total score & No. of patients (\%) & 5-year OS (\%) \\
\hline High risk & $2 \sim 3$ & 55 & 41.7 \\
Intermediate & 1 & 155 & 68.4 \\
Low risk & 0 & 118 & 78.8 \\
\hline
\end{tabular}

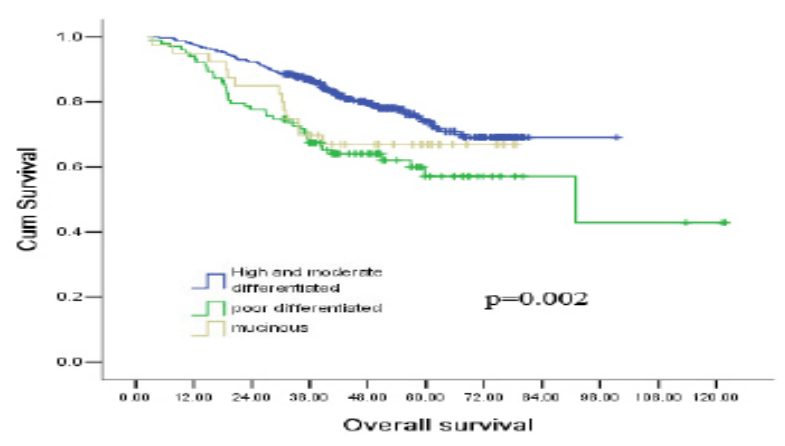

Figure 1. Overall Survival of Patients According to Histology Grade

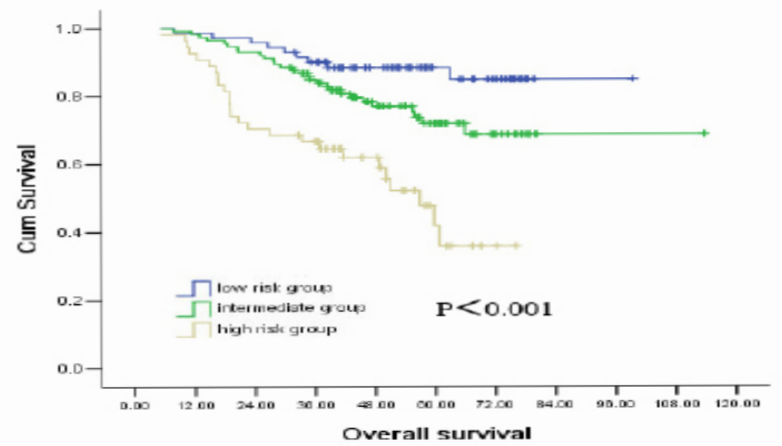

Figure 2. Overall Survival of Patients According to the Prognostic Model in Study Group

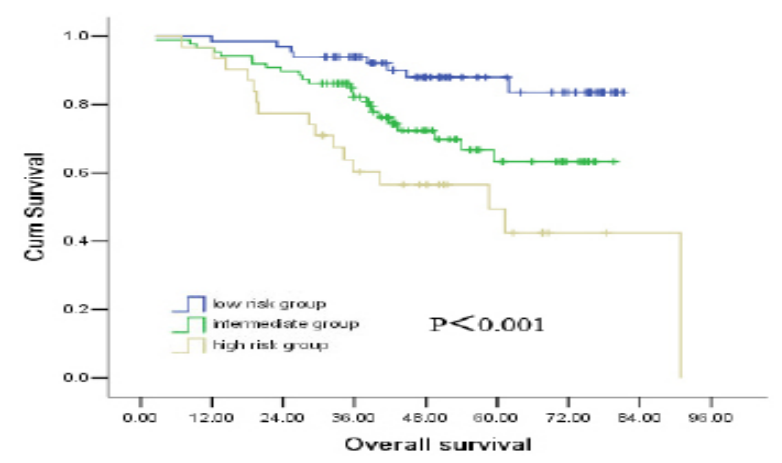

Figure 3. Overall Survival of Patients According to the Prognostic Model in Validation Group

group. The OS (median and range) of patients in low risk group, intermediate group and high risk group were 50.1 (11.9 86.2) months, 41.8 (4.4 79.6) months and 38.3 (5.9 80.9) months, respectively. Kaplan-Meier survival curves were significantly different, shown in Table 4 and Figure 2.

\section{Validation of the prognostic model}

The prognostic model was then validated in an independent patients group whose characteristics were also shown in Table 1.65 patients were in low risk group, 106 patients were in intermediate group and 49 patients were in high risk group, and the OS (median and range) were 54.6 (8.1 94.4.2) months, 48.8 (7.8 84.6) months and 40.4 (5.3 76.3) months, respectively. Consist with the study group, our prognostic model stratified patients in validation group with significantly different survivals (Figure 3). 


\section{Discussion}

In the present study we retrospectively analyzed 548 patients and the main findings were the following: 1) histological grade, preoperative CEA levels and NLR were independent prognostic factors in stage III colon cancer; 2) a prognostic model based on these three factors could effectively indentify patients who were with a 5-year survival rate as $78.8 \%$ and those as $41.7 \%$. Since the three factors are routinely available, they may worth attention and potentially can be applied widely.

The strength of this study relies on the following points. First, a considerable amount of patients were included, and an independent patients group was also involved to validate the model. Second, all the patients involved were with stage III colon cancer and received standard care, the biases were thus minimized. Third, the observed survival rates in the high risk group and low risk group $(41.7 \%$ and $78.8 \%$, respectively) divided by the model showed high accordance with previously reported (Wong et al., 2011).

We are, of course, aware of the limitations of this study. The most important one is that the retrospective nature of this study limits its reliability. And some important parameters, such as venous invasion and perineural invasion, were not documented in some patients' pathological report thus those factors were not included in the study. We also excluded patients accepted emerging surgery since the unprepared surgery and conditions may bring bias to the results. As a logical result, the conclusion in this study cannot be applied to patients who experience an emergency presentation of colon cancer, which happens to about $9 \%$ to $30 \%$ patients (Gunnarsson et al, 2011).

Although $\mathrm{T}$ stage and positive lymph node number were significant prognostic factors in univariate analysis, they lost their influence in multivariate analysis. The possible reason might be that CEA levels were correlated with both $\mathrm{T}$ stage and positive lymph node number in colon cancer (Ma et al., 2006). It was the relevancy that might disappear the impact of $\mathrm{T}$ stage and positive lymph node number in multivariate analysis.

In recent years, people became more and more aware that successful tumors possess the ability to transform the hosts (Hanahan et al., 2000), among those abilities were the tumor-promoting inflammation and immune response (Hanahan et al., 2011)- both elevated neutrophil and decreased lymphocyte were involved in those processes. Indeed, NLR was reported as a unfavorable prognostic factor in many cancers, including breast cancer (Al Murri et al., 2007), colorectal cancer (Chua et al., 2011; Kwon et al., 2012; Mallappa et al., 2013) and lung cancer (Cedres et al., 2012). Neutrophils could promote tumor invasion and metastasis by contributing to angiogenesis and releasing circulating growth factors (Halazun et al., 2009). Lymphocytes, on the other hand, were reported as play a key role in cytotoxic cell death and could inhibit proliferation and metastasis of tumor cells (Ferradini et al., 1991). All of these could serve as the mechanism underlying the significant role of NLR found in our study.

Many genes and proteins had been uncovered as participated in the development and metastasis of colon cancer, and those genes and proteins also showed correlation with OS of stage III colon cancer patients (Bertagnolli et al., 2009; Ogino et al., 2009; Ogino et al., 2012; Belt et al., 2012). Indeed, the knowledge about those biomacromolecule provide us in-depth understanding about cancers. However, these knowledge should not overshadow the importance of basic clinic-pathologic feathers, such as histological grade. What's more, the clinic-pathologic feathers should be taken in account when a new molecular marker is going to be studied in order to achieve a more accurate prognostic classification and a better understanding to the markers.

Several target drugs, which have been proved as effective in metastatic colorectal cancer, unfortunately failed to show superiority in adjuvant treatment, such as bevacizumab (Allegra et al., 2011) and cetuximab (Alberts et al., 2012). Many reasons have been proposed to explain. Although the author of the study claimed the failure was unlikely to be caused by patients' selection (Alberts et al., 2012), it is still reasonable to advice further investigators of clinical trials to keep a close eye on the three factors found in this study.

In conclusion, our study provide a convenient and powerful prognostic model to stratify stage III colon cancer patients based on histological grade, preoperative CEA levels and NLR. Further prospective study with better design is needed to validate this founding.

\section{References}

Adlard JW, Richman SD, Seymour MT, et al (2002). Prediction of the response of colorectal cancer to systemic therapy. Lancet Oncol, 3, 75-82.

Al Murri AM, Wilson C, Lannigan A, et al (2007). Evaluation of the relationship between the systemic inflammatory response and cancer-specific survival in patients with primary operable breast cancer. Br J Cancer, 96, 891-5.

Alberts SR, Sargent DJ, Nair S, et al (2012). Effect of oxaliplatin, fluorouracil, and leucovorin with or without cetuximab on survival among patients with resected stage III colon cancer: a randomized trial. JAMA, 307, 1383-93.

Allegra CJ, Yothers G, O'Connell MJ, et al (2011). Phase III trial assessing bevacizumab in stages II and III carcinoma of the colon: results of NSABP protocol C-08. J Clin Oncol, 29, 11-6.

Barton MK (2012). Oxaliplatin in the adjuvant treatment of colon cancer. CA Cancer J Clin, 62, 3-4.

Belt EJ, Brosens RP, Delis-van Diemen PM, et al (2012). Cell cycle proteins predict recurrence in stage II and III colon cancer. Ann Surg Oncol, 19, 682-92.

Bertagnolli MM, Niedzwiecki D, Compton CC, et al (2009). Microsatellite instability predicts improved response to adjuvant therapy with irinotecan, fluorouracil, and leucovorin in stage III colon cancer: Cancer and Leukemia Group B Protocol 89803. J Clin Oncol, 27, 1814-21.

Cassidy J, Clarke S, Diaz-Rubio E, et al (2008). Randomized phase III study of capecitabine plus oxaliplatin compared with fluorouracil/folinic acid plus oxaliplatin as first-line therapy for metastatic colorectal cancer. J Clin Oncol, 26, 2006-12.

Cedres S, Torrejon D, Martinez A, et al (2012). Neutrophil to lymphocyte ratio (NLR) as an indicator of poor prognosis in stage IV non-small cell lung cancer. Clin Transl Oncol, 14, 864-9. 
Chiang SF, Hung HY, Tang R, et al (2012). Can neutrophil-tolymphocyte ratio predict the survival of colorectal cancer patients who have received curative surgery electively? Int J Colorectal Dis, 27, 1347-57.

Chua W, Charles KA, Baracos VE, et al (2011). Neutrophil/ lymphocyte ratio predicts chemotherapy outcomes in patients with advanced colorectal cancer. Br J Cancer, $\mathbf{1 0}$ 1288-95.

Ferradini L, Miescher S, Stoeck M, et al (1991). Cytotoxic potential despite impaired activation pathways in $\mathrm{T}$ lymphocytes infiltrating nasopharyngeal carcinoma. Int $J$ Cancer, 47, 362-70

Giraldez MD, Lozano JJ, Cuatrecasas M, et al (2013). Geneexpression signature of tumor recurrence in patients with stage II and III colon cancer treated with 5' fluoruracil-based adjuvant chemotherapy. Int J Cancer. 132, 1090-7

Gunnarsson H, Holm T, Ekholm A, et al (2011). Emergency presentation of colon cancer is most frequent during summer. Colorectal Dis, 13, 663-8.

Halazun KJ, Hardy MA, Rana AA, et al (2009). Negative impact of neutrophil-lymphocyte ratio on outcome after liver transplantation for hepatocellular carcinoma. Ann Surg, 250, 141-51.

Hanahan D, Weinberg RA (2000). The hallmarks of cancer. Cell, 100, 57-70.

Hanahan D, Weinberg RA (2011). Hallmarks of cancer: the next generation. Cell, 144, 646-74.

Kuebler JP, Wieand HS, O'Connell MJ, et al (2007). Oxaliplatin combined with weekly bolus fluorouracil and leucovorin as surgical adjuvant chemotherapy for stage II and III colon cancer: results from NSABP C-07. J Clin Oncol, 25, 2198-204.

Kwon HC, Kim SH, Oh SY, et al (2012). Clinical significance of preoperative neutrophil-lymphocyte versus plateletlymphocyte ratio in patients with operable colorectal cancer. Biomarkers, 17, 216-22.

Ma CJ, Hsieh JS, Wang WM, et al (2006). Multivariate analysis of prognostic determinants for colorectal cancer patients with high preoperative serum CEA levels: prognostic value of postoperative serum CEA levels. Kaohsiung J Med Sci, 22, 604-9.

Mallappa S, Sinha A, Gupta S, et al (2013). Preoperative neutrophil lymphocyte ratio greater than five is a prognostic factor for recurrent colorectal cancer. Colorectal Dis, 15, 323-8

Moertel CG, Fleming TR, Macdonald JS, et al (1990). Levamisole and fluorouracil for adjuvant therapy of resected colon carcinoma. $N$ Engl J Med, 322, 352-8.

O'Connell JB, Maggard MA, Ko CY (2004). Colon cancer survival rates with the new American Joint Committee on Cancer sixth edition staging. J Natl Cancer Inst, 96, 1420-5.

Ogino S, Meyerhardt JA, Irahara N, et al (2009). KRAS mutation in stage III colon cancer and clinical outcome following intergroup trial CALGB 89803. Clin Cancer Res, 15, 7322-9.

Ogino S, Shima K, Meyerhardt JA, et al (2012). Predictive and prognostic roles of BRAF mutation in stage III colon cancer: results from intergroup trial CALGB 89803. Clin Cancer Res, 18, 890-900.

Peng Y, Wang L, Gu J, et al (2013). Elevated preoperative carcinoembryonic antigen (CEA) and Ki67 is predictor of decreased survival in IIA stage colon cancer. World J Surg, 37, 208-13

Qiu HB, Zhang LY, Li YF, et al (2011). Ratio of metastatic to resected lymph nodes enhances to predict survival in patients with stage III colorectal cancer. Ann Surg Oncol, 18, 1568-74.

Takagawa R, Fujii S, Ohta M, et al (2008). Preoperative serum carcinoembryonic antigen level as a predictive factor of recurrence after curative resection of colorectal cancer. Ann Surg Oncol, 15, 3433-9.

Wong JC, Chan SK, Schaeffer DF, et al (2011). Absence of MMP2 expression correlates with poor clinical outcomes in rectal cancer, and is distinct from MMP1-related outcomes in colon cancer. Clin Cancer Res, 17, 4167-76. 